\title{
Broadband Metamaterial Solar Absorbers Based on the Earth First-Rate Temperature-Insensitivity Metals for High Temperature Energy Harvesting
}

Elhoussaine Ouabida ( $\nabla$ elhoussaine.ouabida@uit.ac.ma )

Ibn Tofail University: Universite Ibn Tofail https://orcid.org/0000-0003-4468-6676

\section{Research Article}

Keywords: Solar absorbers, Solar metamaterial absorbers, photons management systems, broadband absorbers, high temperature applications.

Posted Date: January 31st, 2022

DOI: https://doi.org/10.21203/rs.3.rs-1279819/v1

License: (9) This work is licensed under a Creative Commons Attribution 4.0 International License.

Read Full License 


\title{
Broadband metamaterial solar absorbers based on the Earth first-rate temperature-insensitivity metals for high temperature energy harvesting
}

\author{
Elhoussaine Ouabida ${ }^{*}$ \\ $1^{*}$ Advanced Systems Engineering Laboratory, National School of \\ Applied Sciences, Ibn Tofail University, University Campus, Po \\ Box 242, Kenitra, Morocco.
}

\author{
Corresponding author(s). E-mail(s): \\ elhoussaine.ouabida@uit.ac.ma;
}

\begin{abstract}
Artificially engineered solar absorbers, known as metamaterial solar absorbers (MSA), are yielding new opportunities for designing new photons management systems. Further, the noble metals are indispensable in creating MSA owing to their plasmonic resonance in a desired spectral region. Nevertheless, in high temperature applications, the noble metals suffer from low melting points that make their uses largely impractical. In this work, the solution of using four of the Earth first-rate temperatureinsensitivity metals in a proposed MSA is investigated. The proposed MSA structure is composed of high melting point metals (HMPM), deposited on a magnesium fluoride $\left(\mathrm{MgF}_{2}\right)$ dielectric spacer and a tungsten continuous plate. Meanwhile, owing to the dependence of MSA properties on structural parameters rather than band structure or chemistry, a geometrical optimization of the proposed absorbers is reported. Furthermore, the proposed MSA performance is investigated by computing the absorption spectrums. Results show relatively long bandwidths in the visible and near-infrared regimes. Besides, an underlying mechanism of the absorption enhancement with the corresponding electromagnetic field distributions are elucidated in detail. The calculated results indicate that the proposed MSA present wide bandwidths owing to the excitation of resonance modes of surface plasmons, dipolar interactions and cavity modes. Equally important, absorption measurements under
\end{abstract}


wide polarization angles show polarization-insensitive high absorption at higher wavelengths, which is among the most important factors for an ideal solar absorber. The conclusion of this investigation is undoubtedly important, specifically that the proposed HMPM-based MSA would be the best choice for energy harvesting in high temperature applications.

Keywords: Solar absorbers, solar metamaterial absorbers, photons management systems, broadband absorbers, high temperature applications.

\section{Introduction}

Owing to the increases in the global energy request of up to $1 \mathrm{GW} /$ day [1], research in efficient and low-cost energy production tools is currently taking place at a breathtaking pace. Meanwhile, a clean and abundant energy source as the Sun makes the solar energy promising for the next generation of energy production technologies. Thus, solar photovoltaic [2, 3], thermophotovoltaic [4-6], and solar thermal $[7,8]$ are typical approaches that have been widely investigated and reported. However, with the fails of covering the full spectrum shown by photovoltaic approach owing to the semiconductor bandgap limit, the need for an ideal energy harvesting approach has become more acute. Alternatively, converting sunlight to energy within the full spectrum solar energy can be achieved by thermophotovoltaic and solar thermal approaches.

The emerging field of artificially engineered materials, known as metamaterials, has attracted much attention in the last years for potential use in thermophotovoltaic systems (TPVS) and solar thermal approach [9-14]. By demonstrating interesting electromagnetic properties not found in naturally occurring materials, the metamaterial solar absorbers (MSA) have opened a new avenue for solar energy harvesting. The first experimental demonstration of MSA can be dated back to 2008 [15]. From then on, research in this field has been extensively employed for proposing new structures and exploring new materials, most prominently in a context of controlling the trade-off that exists between absorption bandwidth and absorption strength. This control can be obtained by adjusting the structural parameters of the metamaterial absorber structure. For instance, authors in [14] studied the influence of structural parameters of a metamaterial absorber structured via a core-shell nanocone made of silica core and tungsten shell. On the one hand, ideal MSA should present several bands of the spectral absorption. For instance, Bhargav Appasani proposed in [12] a seven-band metamaterial absorber with slotted flower-shaped resonator (S-FSR) on InSb dielectric substrate. Additionally, a metamaterial absorber with three absorption peaks at the terahertz regime is investigated in [16]. On the other hand, resonances and coupling modes are other ways to tack with the metamaterial absorber problem. For instance, a metamaterial absorber based on local surface plasmon resonance excited 
between molybdenum disulfide $\left(\mathrm{MoS}_{2}\right)$ and tungsten (W) elliptical arrays is proposed in [17]. Meanwhile, in addition to these exciting electromagnetic properties, metamaterial absorbers exhibit other salient properties as performances shifting by using external stimuli as gravity field regulation, temperature, and voltage $[12,18]$. Metamaterials found also applications in other fields as biomedical for sensing both the temperature and the refractive index [19]. Unfortunately, most of the previous studies on MSA use silver, gold and other noble metals that suffer from low melting points [15]. Further, the operation temperature of such system as TPVS is usually passing the melting points of the used noble metals. Hence, the currently main challenge is how to design high-temperature MSA and future work should be focused on designing and fabricating high-temperature resistant MSA. Therefore, new studies that arouse considerable interest on temperature resistant MSA will redesign and optimize various models prior to experiment and must be encouraged.

In this work, we report on the solution of using four of the Earth first-rate high melting point metals (HMPM) in a proposed structure of metamaterial solar absorbers. HMPM are deposited on a magnesium fluoride $\left(\mathrm{MgF}_{2}\right)$ dielectric spacer and a tungsten continuous metallic plate (Figure 1). The HMPM are tungsten (W), rhenium (Re), tantalum (Ta) and molybdenum (Mo). On the one hand, W, Re, Ta, and Mo are chosen owing to the outstanding high temperature insensitivity. For the dielectric spacer, the $\mathrm{MgF}_{2}$ is chosen due to the better thermal expansion coefficient (TEC). Its high TEC has made the $\mathrm{MgF}_{2}$ the best candidate in high temperature applications where other dielectrics suffer from the thermal cracking possibility. On the other hand, because of the large number of structural parameters influencing the spectral absorption, the structural parameters resulting in near unity absorption are explored. Besides, for each HMPM, absorption spectrums are measured over the spectral region of interests composed from UV to the mid near-infrared (NIR) regimes $(0.3 \mu \mathrm{m}-2 \mu \mathrm{m})$. Moreover, the resonance properties (absorption strength and resonance wavelength) and the electromagnetic field distribution are investigated. Finally, the angular behavior of the proposed MSA is examined under the change of the polarization angle. 


\section{Material and methods}

The metamaterial solar absorbers (MSA) exhibit the most salient feature of electromagnetic properties dependence on the structural parameters rather than the band structure or chemistry. Hence, it is insightful for us to adjust the structural parameters to effectively control the absorption spectrum. Figure 1 depicts the structure of the proposed MSA based on HMPM. The proposed structure was designed using three layers with specific structural parameters. The top layer was a patterned HMPM by trapezoid grating and characterized by four parameters $\left(W_{B}, W_{T}, G_{H}\right.$ and $P$ ). The intermediate medium was an $\mathrm{MgF}_{2}$ dielectric spacer with $S_{H}$ thickness. The bottom layer was a tungsten continuous metallic plate working as an eliminator of the incident electromagnetic wave. This can be obtained by adjusting its thickness $T_{H}$ so it can be opaque within the spectral region of interests. Hence, finite element method (FEM) simulation was performed to numerically calculate the spectral absorption while optimizing the structural parameters. First, the developed FEM model results were validated by reproducing the previous experimental investigation in [9]. Then, the developed FEM model was modified to solve the Helmholtz equation derived from Maxwell's equations in our situation. The plane wave source is used in the FEM model to simulate the electromagnetic wave, which is configured to reach the structure with a small nonzero oblique polarization angle of $\theta=8^{\circ}$ resulting in a larger in-plane wavevectors $K_{\text {inc }} \neq 0$. Both time and space were discretized in a $2 \mathrm{D}$ boxes (along $x$ and $y$ axes) with propagation along the $y$ axis. The boxes corresponded to the air, the dielectric spacer and to the continuous metallic plate. The HMPM were periodically designed above the box of the $\mathrm{MgF}_{2}$ dielectric spacer. For the HMPM, it is clear from Table 1 that the metals with the high temperature insensitivity are tungsten (W), rhenium (Re), tantalum ( Ta) and molybdenum (Mo). Hence, W, Re, Ta, and Mo were used advantageously in designing high-efficiency MSA. Further, all the materials of the unit cell were defined in the FEM model using the corresponding optical properties obtained from Palik's data [20]. The whole structure was supposed to be repeated periodically along the $x$ direction. Furthermore, a mesh generation technique was used along the entire boxes of the structure to generate mesh elements. Since the elements size significantly affects the convergence results, the mesh size was validated with previous experimental investigation. We want to stress that it is with this validity of the developed FEM model in mind that the HMPM were investigated in this work. The developed FEM model was used to compute spectral absorption of the HMPM by using the formula $A(\lambda)=1-R(\lambda)-T(\lambda)$. 


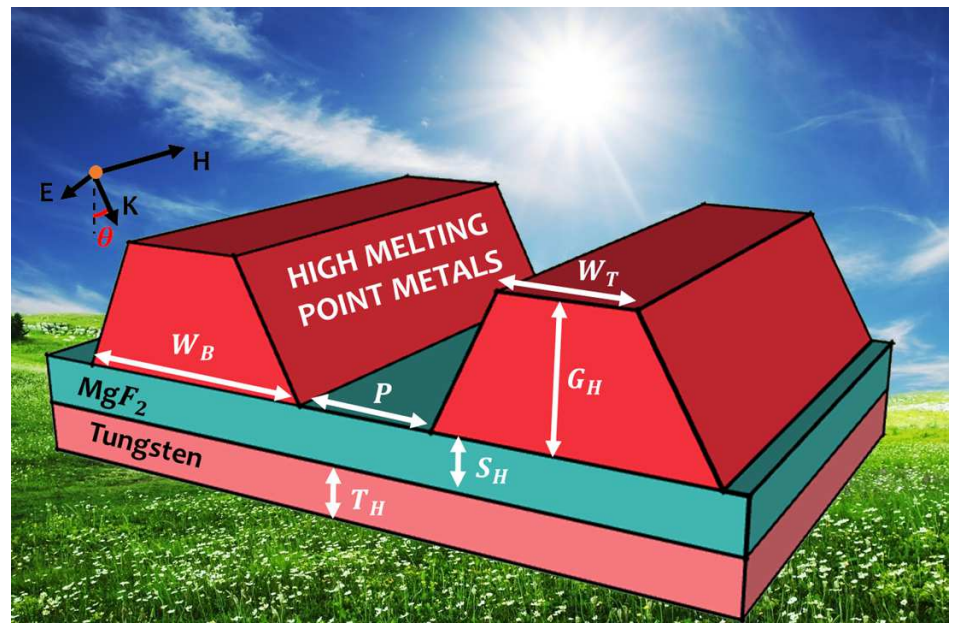

Figure 1 Structure schematic for the proposed HMPM based MSA. $K$ represents the incident wavevector, $E$ is for the electric field for transverse magnetic (TM) incidence, and the $\theta$ polarization angle.

Table 1 Melting temperatures of the elements in the standard state at 1 atm in degree Celsius ${ }^{\circ} \mathrm{C}$ from [21].

\begin{tabular}{|c|c|c|c|c|c|c|c|c|c|c|c|c|c|c|c|c|c|}
\hline $\begin{array}{c}\mathrm{H} \\
-259\end{array}$ & & & & & & & & & & & & & & & & & $\begin{array}{c}\mathrm{He} \\
-272\end{array}$ \\
\hline $\begin{array}{r}\mathrm{Li} \\
181\end{array}$ & $\begin{array}{c}\mathrm{Be} \\
1287\end{array}$ & & & & & & & & & & & $\begin{array}{c}\text { B } \\
2075\end{array}$ & $\begin{array}{c}\mathrm{C} \\
3500\end{array}$ & $\begin{array}{c}\mathrm{N} \\
-210\end{array}$ & $\begin{array}{c}\mathrm{O} \\
-219\end{array}$ & $\begin{array}{c}F \\
-219\end{array}$ & $\begin{array}{c}\mathrm{Ne} \\
-249\end{array}$ \\
\hline $\begin{array}{c}\mathrm{Na} \\
98 \\
\end{array}$ & $\begin{array}{l}\mathrm{Mg} \\
650 \\
\end{array}$ & & & & & & & & & & & $\begin{array}{c}\mathrm{Al} \\
660\end{array}$ & $\begin{array}{c}\mathrm{Si} \\
1414\end{array}$ & $\begin{array}{c}\mathrm{P} \\
44 \\
\end{array}$ & $\begin{array}{c}\mathrm{S} \\
115 \\
\end{array}$ & $\begin{array}{c}\mathrm{Cl} \\
-102 \\
\end{array}$ & $\begin{array}{c}\mathrm{Ar} \\
-189 \\
\end{array}$ \\
\hline K & $\mathrm{Ca}$ & $\mathrm{Sc}$ & $\mathrm{Ti}$ & V & $\mathrm{Cr}$ & $\mathrm{Mn}$ & $\mathrm{Fe}$ & Co & $\mathrm{Ni}$ & $\mathrm{Cu}$ & $\mathrm{Zn}$ & $\mathrm{Ga}$ & $\mathrm{Ge}$ & As & $\mathrm{Se}$ & $\mathrm{Br}$ & $\mathrm{Kr}$ \\
\hline Cs & $\mathrm{Ba}$ & $\mathrm{Lu}$ & $\mathrm{Hf}$ & $\mathrm{Ta}$ & W & Re & Os & Ir & $\mathrm{Pt}$ & $\mathrm{Au}$ & $\mathrm{Hg}$ & $\mathrm{Tl}$ & $\mathrm{Pb}$ & $\mathrm{Bi}$ & Po & At & $\mathrm{Rn}$ \\
\hline 29 & 727 & 1663 & 2233 & 3017 & 3422 & 3186 & 3033 & 2446 & 1768 & 1064 & -39 & 304 & 327 & 271 & 254 & 302 & -71 \\
\hline $\begin{array}{l}\text { Fr } \\
27\end{array}$ & $\begin{array}{c}\mathrm{Ra} \\
696\end{array}$ & $\begin{array}{c}\mathrm{Lr} \\
1627\end{array}$ & $\mathrm{Rf}$ & $\mathrm{Db}$ & $\mathrm{Sg}$ & $\mathrm{Bh}$ & $\mathrm{Hs}$ & $\mathrm{Mt}$ & Ds & Rg & $\mathrm{Cn}$ & $\mathrm{Nh}$ & $\mathrm{Fl}$ & Mc & $\mathrm{Lv}$ & $\mathrm{Ts}$ & $\mathrm{Og}$ \\
\hline
\end{tabular}

\section{Results and Discussion}

\subsection{Structural parameters optimization}

All the structural parameters were varied from $10 \mathrm{~nm}$ to $1 \mu \mathrm{m}$ with a step of $10 \mathrm{~nm}$. Since the contact widths of the HMPM with the dielectric spacer and air affect the resonance depth, the investigated parameters in the first set of experiments were the grating bottom width $W_{B}$, the top width $W_{T}$ and the grating height $G_{H}$. In the second set of experiments, the investigated parameter was the grating periodicity $P$. This parameter is important to understand the underlying physics interaction between two grating. The investigated parameter in the third set of experiments was the thickness $T_{H}$ of the tungsten metallic plate, which is vital for blocking all the incidents electromagnetic 
waves by making it opaque within the spectral region of interests. In the last set of experiments, the investigated parameter was the dielectric spacer thickness $S_{H}$. In this way, the overall performance in terms of absorption spectrum was enhanced significantly. The optimized structural parameters of the proposed structure with larger absorption spectrum bandwidths are set in Table 2.

Table 2 The optimized structural parameters in $n m$ of the proposed MSA.

\begin{tabular}{|c|c|c|c|c|c|c|}
\hline & $\begin{array}{r}\mathrm{HM} \\
W_{B}\end{array}$ & $W_{T}$ & $G_{H}$ & $P$ & $\begin{array}{c}\text { Dielectrid } \\
\text { spacer } \\
S_{H}\end{array}$ & $\begin{array}{c}\text { Tungsten } \\
\text { plate } \\
T_{H} \\
\end{array}$ \\
\hline Tungsten (W) & 300 & 80 & 720 & 170 & \multirow{4}{*}{50} & \multirow{4}{*}{200} \\
\hline Rhenium (Re) & 280 & 100 & 910 & 260 & & \\
\hline Tantalum (Ta) & 200 & 100 & 690 & 220 & & \\
\hline Molybdenum (Mo) & 300 & 100 & 950 & 250 & & \\
\hline
\end{tabular}

\subsection{Spectral absorption}

The premise to efficient harvesting of solar energy is near unity absorption of solar spectrum. Hence, in this section, we are in a position to discuss the absorption spectrums of the proposed MSA with the optimized structural parameters in Table 2. Firstly, Figure 2 (W) shows the absorption spectrum of the proposed MSA with tungsten as grating metal. The absorption spectrum was measured at different wavelengths with a polarization angle of $\theta=8^{\circ}$. The absorption of the W-based MSA was higher than $90 \%$ in a relatively long bandwidth. After the geometrical optimization, the bandwidth with absorption higher than $90 \%$ was greatly expanded to $1390 \mathrm{~nm}$ long (at $300 \mathrm{~nm} \leq \lambda \leq 1690$ $n m$ ). Besides, Figure $2(\mathrm{~W})$ also illustrates that the proposed W-based MSA was highly efficient in the visible region with a maximum absorption of 0.9997 at $\lambda=375 \mathrm{~nm}$. Secondly, Figure 2 (Re) displays in the same way the absorption spectrum of the proposed MSA with rhenium as grating metal. The absorption of the Re-based MSA was higher than $90 \%$ in a bandwidth of 1440 $\mathrm{nm}$ long (at $300 \mathrm{~nm} \leq \lambda \leq 1740 \mathrm{~nm}$ ). Further, absorption spectrum of the Re-based MSA was characterized to be higher than $70 \%$ over the all-spectral region of interests. Equally important, the results of Figure 2 (Re) also show that a maximum absorption of 0.9994 was observed at $\lambda=1200 \mathrm{~nm}$ indicating that the proposed Re-based MSA is highly efficient in the NIR region. Thirdly, Figure 2 (Ta) similarly exhibits the absorption spectrum of the proposed MSA with tantalum as grating metal. The absorption of the Ta-based MSA was higher than $90 \%$ in a bandwidth of $720 \mathrm{~nm}$ long (at $300 \mathrm{~nm} \leq \lambda \leq 1020 \mathrm{~nm}$ ). Besides, Ta-based MSA was highly efficient in the visible region with a maximum absorption of 0.9984 at $\lambda=420 \mathrm{~nm}$. Finally, Figure 2 (Mo) depicts the characterized absorption spectrum of the proposed MSA with molybdenum as 
grating metal, measured at different wavelengths in the same above-mentioned conditions. The absorption of the Mo-based MSA was higher than $90 \%$ in a bandwidth of $1200 \mathrm{~nm}$ long (at $300 \mathrm{~nm} \leq \lambda \leq 1500 \mathrm{~nm}$ ). Furthermore, the results of Figure 2 (Mo) also show that a maximum absorption of 0.9999 was observed at $\lambda=330 \mathrm{~nm}$ indicating that the proposed Mo-based MSA is also highly efficient in the visible region.
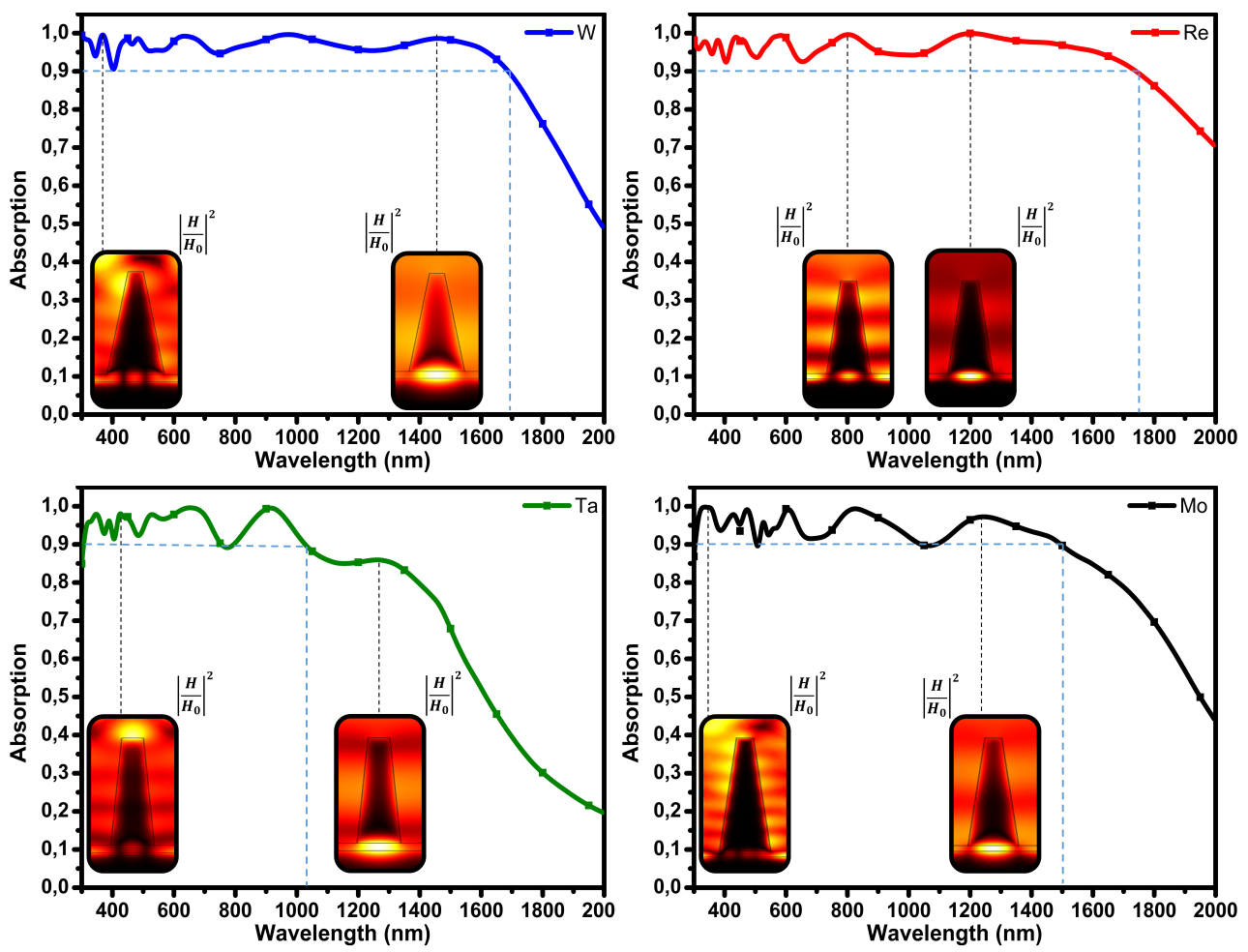

Figure 2 Absorption spectrums of the proposed MSA. The insets show the magnetic field intensity distribution at $\lambda=375 \mathrm{~nm}$ and $\lambda=1455 \mathrm{~nm}$ for $\mathrm{W}$, at $\lambda=795 \mathrm{~nm}$ and $\lambda=1200$ $\mathrm{nm}$ for Re, at $\lambda=420 \mathrm{~nm}$ and $\lambda=1260 \mathrm{~nm}$ for Ta, and at $\lambda=330 \mathrm{~nm}$ and $\lambda=1245 \mathrm{~nm}$ for Mo.

On the whole, we can say that the bandwidths of all the proposed HMPMbased MSA with overall absorption rate of more than $90 \%$ were clearly demonstrated. These larger bandwidths are vital for improving the performance of solar absorbers. At the same time, a significant increment was observed in the performance of the proposed Re-based MSA with the widest bandwidth compared to the other proposed MSA. Next, we will analyze why the proposed MSA present such wide bandwidths within the spectral region of interests. 


\subsection{Underlying mechanisms for high broadband solar absorption}

Emphasis was put on three set of experiments for exhibiting the influence of the parameters with the most important effects on both absorption bandwidth and strength. Based on our findings, parameters with significant effects on performances were the grating bottom width $W_{P}$, the grating period $P$, and the $\mathrm{MgF}_{2}$ spacer thickness $S_{H}$. Especially, the focus was made on analyzing the influence of these parameters' variation on the strength and resonance wavelength of two absorption peaks $\mathrm{AP}_{1}$ and $\mathrm{AP}_{2}$. On the one hand, $\mathrm{AP}_{1}$ is characterized by being the absorption peak with the maximum strength within the all-spectral region of interests. On the other hand, $\mathrm{AP}_{2}$ is the last absorption peak with the maximum strength beyond the one the absorption strength is decreased to values under $90 \%$. The shift effect on $\mathrm{AP}_{1}$ and $\mathrm{AP}_{2}$ by $W_{B}, P$, and $S_{H}$ variations can be used to get larger bandwidths.

Firstly, we discuss the $W_{B}$ variation effect on the absorption performances of the proposed MSA. As can be seen from Figure 3, the absorption strength was greatly enhanced by reaching $W_{B}=300 \mathrm{~nm}$ for tungsten (W), $W_{B}=280$ $\mathrm{nm}$ for rhenium (Re), $W_{B}=200 \mathrm{~nm}$ for tantalum (Ta) and $W_{B}=300 \mathrm{~nm}$ for molybdenum (Mo). Based on our findings, we can say that $\mathrm{AP}_{1}$ and $\mathrm{AP}_{2}$ were sensitive to the $W_{B}$ variation. This can be explained by the fact that the $\mathrm{AP}_{1}$ and $\mathrm{AP}_{2}$ absorption peaks were excited by the excitation of the surface plasmon modes $([17,22,23])$ at the HMPM surface. At a specific value of $W_{B}$, which corresponds to a specific contact width of the HMPM with the dielectric spacer and air, the coupling effect of incident electromagnetic wave and induced electric field enhances the absorption rates. The magnetic field intensity distributions at the edge of the trapezoid grating shown in the insets of Figure 2 support the above descriptions. 

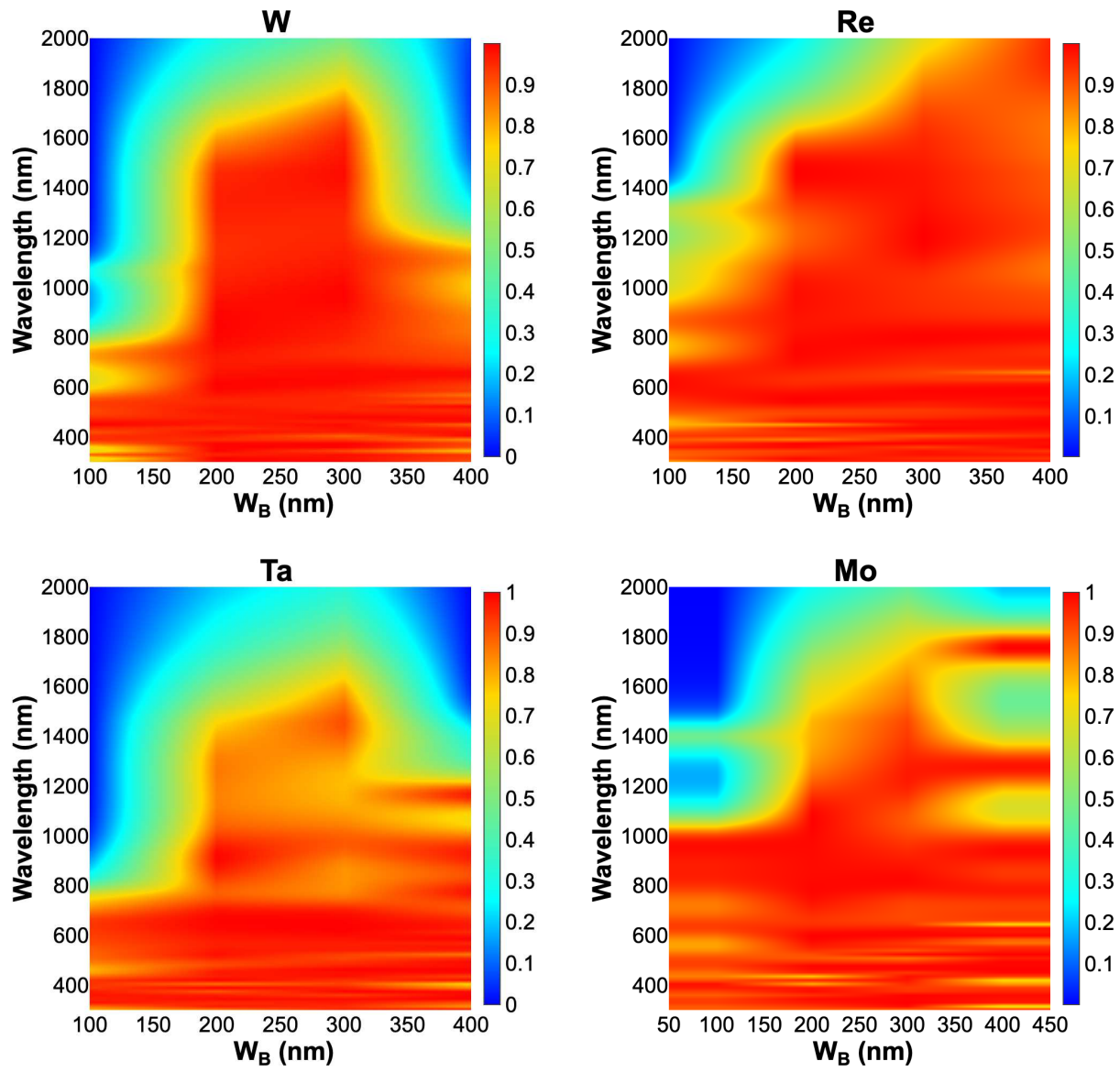

Figure 3 The influence of the $W_{B}$ variation on the performance of the proposed MSA for tungsten (W), rhenium (Re), tantalum (Ta) and molybdenum (Mo). 
Regarding the periodicity set of experiments shown in Figure $4, P$ variation showed a moderate sensitivity of the resonance properties at the range of $150 \mathrm{~nm} \leq P \leq 170 \mathrm{~nm}$ for $\mathrm{W}$, at $200 \mathrm{~nm} \leq P \leq 300 \mathrm{~nm}$ for Re, at 220 $\mathrm{nm} \leq P \leq 250 \mathrm{~nm}$ for Ta and at $150 \mathrm{~nm} \leq P \leq 250 \mathrm{~nm}$ for Mo. At these ranges, dipolar interaction modes $([24,25])$ were excited between two grating and enhanced the absorption strength. For instance, the inset of the magnetic field intensity distribution in Figure $2(\mathrm{Re})$ at $\lambda=795 \mathrm{~nm}$ supports the above description. Beyond these ranges, $\mathrm{AP}_{1}$ and $\mathrm{AP}_{2}$ were highly sensitive to the $P$ variation and showed absorption strength under $90 \%$.
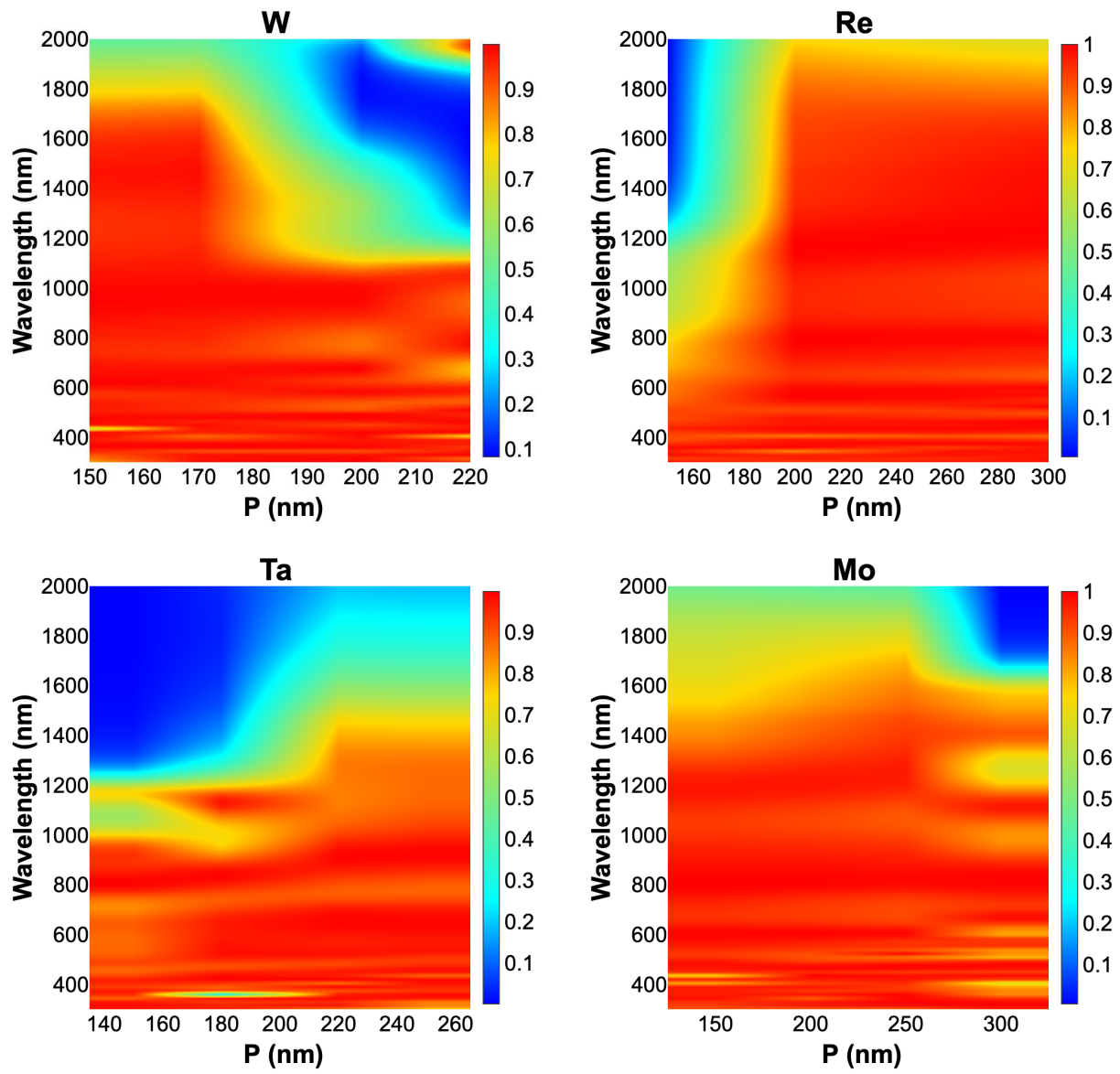

Figure 4 The influence of the $P$ variation on the performance of the proposed MSA for tungsten (W), rhenium (Re), tantalum (Ta) and molybdenum (Mo). 
In the last set of experiments, computed results in Figure 5 of the $\mathrm{MgF}_{2}$ thickness showed that the resonance properties of $\mathrm{AP}_{1}$ were almost unchanged with the variation of the $\mathrm{MgF}_{2}$ thickness. The major explication of this is that the excitation settings of the surface plasmon modes are retained. On the other hand, the resonance properties of $\mathrm{AP}_{2}$ were significantly changed. It is found that the absorption strength of $\mathrm{AP}_{2}$ in all the proposed MSA achieved the maximum value when the thickness reached $S_{H}=50 \mathrm{~nm}$. It can be explained by the fact that $\mathrm{AP}_{2}$ is excited by the cavity modes $([26,27])$ between the layers of upper grating and the tungsten lower plate. The insets in Figure 2 show the excitation of the cavity modes at $\lambda=1455 \mathrm{~nm}$ for tungsten, at $\lambda=1200 \mathrm{~nm}$ for rhenium, at $\lambda=1260 \mathrm{~nm}$ for tantalum, and at $\lambda=1245 \mathrm{~nm}$ for molybdenum.
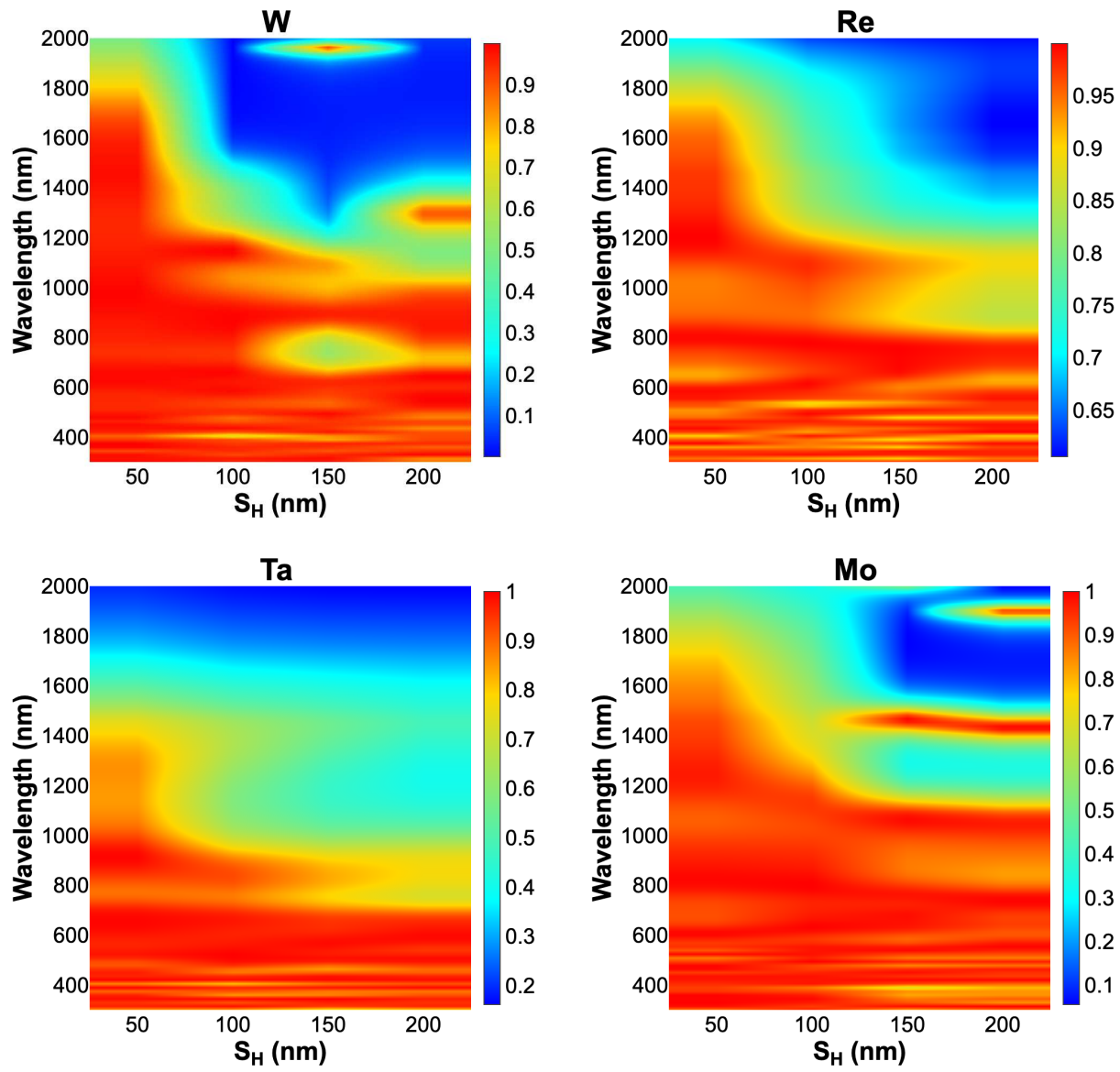

Figure 5 The influence of the $S_{H}$ variation on the performance of the proposed MSA for tungsten $(\mathrm{W})$, rhenium (Re), tantalum (Ta) and molybdenum (Mo). 


\subsection{Angular behavior of the proposed MSA}

Real conditions of random nature of sunlight suggest that the independency of polarization angle is among the most important factors for an ideal solar absorber [14]. Therefore, absorption spectrums of the proposed HMPM-based MSA were investigated at several polarization angles of $\theta=\left[0^{\circ}-50^{\circ}\right]$.
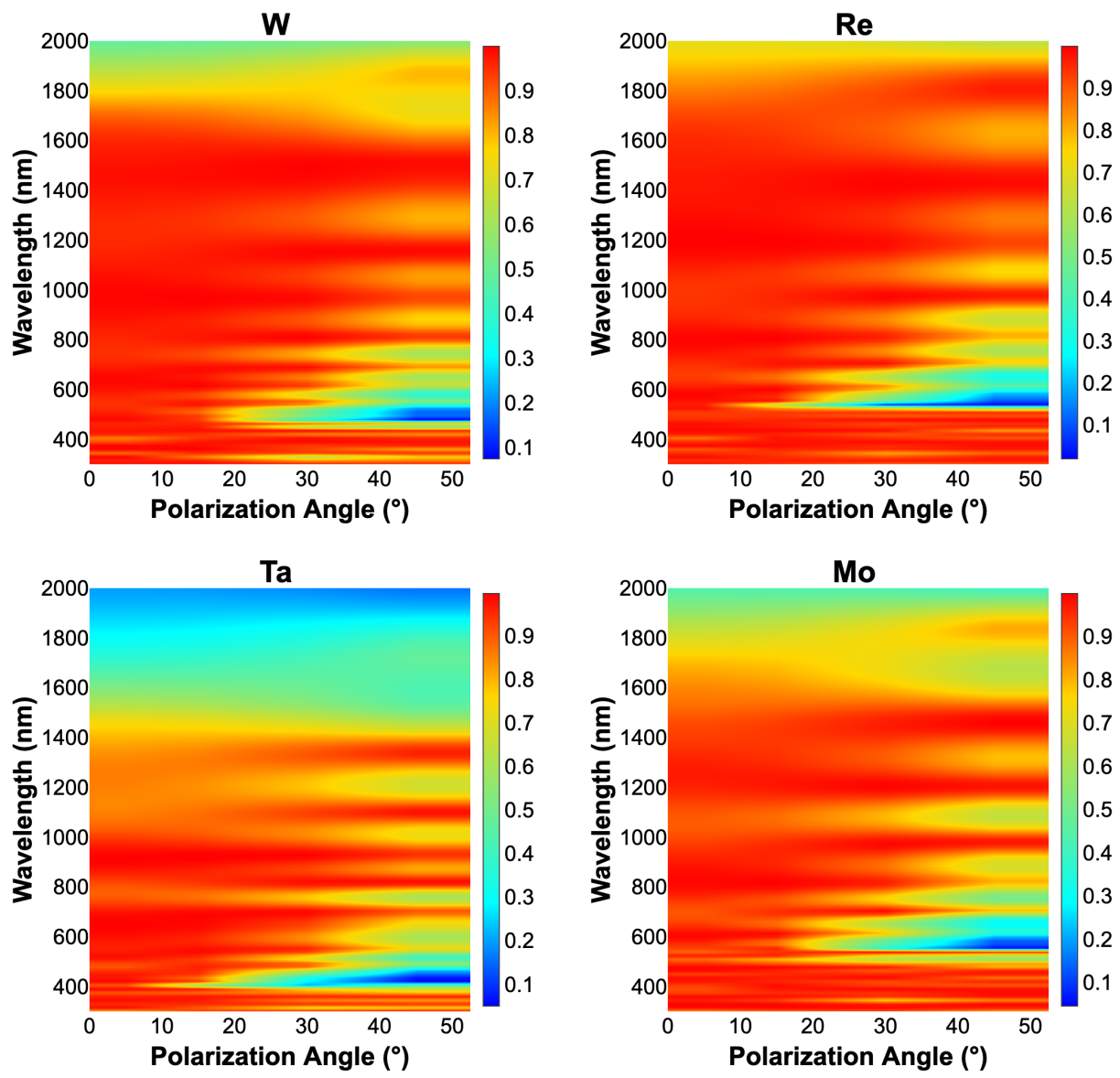

Figure 6 Dependence of absorption spectrums of the proposed MSA on the polarization angle and wavelength for tungsten (W), rhenium (Re), tantalum (Ta) and molybdenum (Mo).

It can be seen from Figure 6 that the absorption strengths at $\lambda \leq 800 \mathrm{~nm}$ of all the proposed MSA are sensitive to the polarization angle. The absorption strengths at $\lambda \leq 800 \mathrm{~nm}$ decreased with increased polarization angles. This can be explained by the fact that, at lower polarization angles, the excitation of the surface plasmon and cavity modes excites the absorption peaks $\mathrm{AP}_{1}$ and $\mathrm{AP}_{2}$. At larger polarization angles, the above-mentioned modes are 
unexcited and absorption strengths decrease. For instance, the analysis of the electromagnetic field distribution at $\lambda=520 \mathrm{~nm}$ of the Re-based MSA showed a higher cavity mode intensity for $\theta=5^{\circ}$ while this mode disappeared at $\theta=45^{\circ}$.

For $\lambda \geq 800 \mathrm{~nm}$ that is more than $70 \%$ of the spectral region of interests, it is found that the absorption strengths of all the proposed HMPM-based MSA are slightly sensitive to the polarization angles. This can be explained by the fact that, with the chosen thickness of the dielectric spacer, the cavity modes are maintained at higher wavelengths within spectral region of interests. On the whole, the measured absorption spectrums show a polarization angle independent behavior at higher wavelengths of all the proposed HMPM-based MSA indicating wide-angle insensitive high absorption.

\section{Conclusion}

In summary, we have proposed and numerically studied the solution of using four of the first-rate temperature-insensitivity metals as periodically grating patterns in a proposed structure of a metamaterial solar absorber (MSA). Summing up the results, the proposed MSA are demonstrated to exhibit bandwidths long with absorption higher than $90 \%$ of $1390 \mathrm{~nm}, 1440 \mathrm{~nm}, 720 \mathrm{~nm}$, and $1200 \mathrm{~nm}$, respectively for tungsten, rhenium, tantalum, and molybdenum. Further, the trade-off between absorption bandwidth and strength is tuned by carefully optimizing the geometrical parameters of the proposed structure. Besides, computed absorption spectrums show wide-angle-insensitive high absorption at higher wavelengths of the spectral region of interests. Our findings suggest that tungsten, rhenium, tantalum, and molybdenum may be widely used in the design of a new class of high temperature solar absorbers for applications in different areas. Several other questions about the HMPM-based MSA as the performance of other 3D shapes remain to be addressed in our future research work.

Acknowledgments. The author would like to thank Professor Liping Wang (School for Engineering of Matter, Transport and Energy, Arizona State University, USA) for his helpful details about his work and for all the replying messages about finding data.

\section{Statements and Declarations}

- Funding: The author declares that no funds, grants, or other support were received during the preparation of this manuscript.

- Conflict of interest/Competing interests: The author has no relevant financial or non-financial interests to disclose. The author declares that he has no conflict of interest. 
- Ethics approval: This research effort is not involving human participants, their data or biological material. In addition, it is not involving animals, their data or biological material: no ethical approval is required.

- Consent to participate: The author gives his consent to participate.

- Consent for publication: The author gives his consent to publish.

- Availability of data and materials: The datasets generated during and/or analysed during the current study are available from the corresponding author on reasonable request.

- Authors' contributions: The author has conceived the idea, designed and simulated the structure, obtained the results, and has written the manuscript.

\section{References}

[1] Wang, D., Wright, M., Elumalai, N. K., Uddin, A. (2016) Stability of perovskite solar cells. Sol. Energy Mater. Sol. Cells 147, 255-275 . https://doi.org/10.1016/j.solmat.2015.12.025

[2] Parida, B., Iniyan, S. , Goic, R. (2011) A review of solar photovoltaic technologies. Renew. Sustain. Energy Rev. 15, 1625-1636 . https://doi.org/10.1016/J.RSER.2010.11.032

[3] Gul, M., Kotak, Y. , Muneer, T. (2016) Review on recent trend of solar photovoltaic technology. Energy Explor. Exploit. 34, 485-526 . https://doi.org/10.1177/0144598716650552

[4] Bauer, T. (2011) Thermophotovoltaics: Basic principles and critical aspects of system design. Springer, Berlin, Heidelberg vol. 7 . https://doi.org/10.1007/978-3-642-19965-3

[5] Hassan, S., Doiron, C. F. , Naik, G. V. (2020) Optimum selective emitters for efficient thermophotovoltaic conversion. Appl. Phys. Lett. 116, 023903 . https://doi.org/10.1063/1.5131367

[6] Shimizu, M., Kohiyama, A. , Yugami, H. (2015) High-efficiency solar-thermophotovoltaic system equipped with a monolithic planar selective absorber/emitter. J. Photonics Energy 5, 053099 . https://doi.org/10.1117/1.JPE.5.053099 
[7] Deng, Z., Zhou, J., Miao, L., Liu, C., Peng, Y., Sun, L., Tanemura, S. (2017) The emergence of solar thermal utilization: solar-driven steam generation. J. Mater. Chem. A 5, 7691-7709 . https://doi.org/10.1039/C7TA01361B

[8] Cao, Y., Hashemian, M., Ayed, H., Shawabkeh, A., Issakhov, A., Wae-hayee, M. (2022) Design-eligibility study of solar thermal helically coiled heat exchanging system with annular dimples by irreversibility concept. Renew. Energy 183, 369-384 . https://doi.org/10.1016/J.RENENE.2021.10.095

[9] Wang, H., Prasad S. V., Mitchell, A., Rosengarten, G., Phelan, P., Wang, L. (2015) Highly efficient selective metamaterial absorber for high-temperature solar thermal energy harvesting. Sol. Energy Mater. Sol. Cells 137, 235-242. https://doi.org/10.1016/j.solmat.2015.02.019

[10] Bendelala, F. , Cheknane, A. (2022) A Transparent Metasurface Absorber/Emitter with High Solar Thermal Transfer Efficiency for Combined Solar/Thermal Conversion Application. Plasmon. 2022 1-9 https://doi.org/10.1007/S11468-021-01580-W

[11] Elsharabasy, A., Bakr, M. , Deen, M. J. (2020) Wide-angle, wide-band, polarization-insensitive metamaterial absorber for thermal energy harvesting. Sci. Rep. 10, 1-10. https://doi.org/10.1038/s41598-020-73368-7

[12] Appasani, B. (2021) Temperature Tunable Seven Band Terahertz Metamaterial Absorber Using Slotted Flower-Shaped Resonator on an InSb Substrate. Plasmonics 16, 833-839. https://doi.org/10.1007/s11468-020-01329-x

[13] Hoque, A. , Islam, M. T. (2020) Numerical Analysis of Single Negative Broadband Metamaterial Absorber Based on Tri Thin Layer Material in Visible Spectrum for Solar Cell Energy Harvesting. Plasmonics 15, 1061-1069 . https://doi.org/10.1007/s11468-020-01132-8

[14] Da, Y. , Xie, M. (2021) Solar Selective Absorbers for High-efficiency Photothermal Conversion via Core-Shell Nanocone Structured Surface. Plasmonics 16, 589-597. https://doi.org/10.1007/s11468-020-01317-1 
[15] Yu, P., Besteiro, L. V., Huang, Y., Wu, J., Fu, L., Tan, H. H., Jagadish, C., Wiederrecht, G. P. Govorov, A. O. Wang, Z. (2019) Broadband Metamaterial Absorbers. Adv. Opt. Mater. 7, 1-32. https://doi.org/10.1002/adom.201800995

[16] Rahmanshahi, M., Noori Kourani, S., Golmohammadi, S., Baghban, H. , Vahed, H. (2021) A Tunable Perfect THz Metamaterial Absorber with Three Absorption Peaks Based on Nonstructured Graphene. Plasmonics 16, 1665-1676. https://doi.org/10.1007/s11468-021-01432-7

[17] Li, J., Chen, X., Yi, Z., Yang, H., Tang, Y., Yi, Y., Yao, W., Wang, J., Yi, Y. (2020) Broadband solar energy absorber based on monolayer molybdenum disulfide using tungsten elliptical arrays. Mater. Today Energy 16, 100390. https://doi.org/10.1016/j.mtener.2020.100390

[18] Tian, X. L., Zhang, H. F. , Kong, X. R. (2020) An Angle-Insensitive Metamaterial Absorber Based on the Gravity Field Regulation. Plasmonics 15, 517-523. https://doi.org/10.1007/s11468-019-01077-7

[19] Appasani, B. (2021) A Hybrid Terahertz Metamaterial Sensor Using a Hexagonal Ring Resonator with Bio-medical Applications. Plasmon. 2021 1-6. https://doi.org/10.1007/S11468-021-01546-Y

[20] PALIK, E. D. (1998) Handbook of Optical Constants of Solids. Academic Press. https://www.sciencedirect.com/book/9780125444224/ handbook-of-optical-constants-of-solids

[21] Lide, D. R. (2005) CRC Handbook of Chemistry and Physics. CRC Press LLC: Boca Raton, FL. (American Chemical Society (ACS)). https://doi.org/10.1021/ja041017a

[22] Maier, S. A. (2007) Plasmonics: Fundamentals and applications. Springer, New York, NY (Springer US). https://doi.org/10.1007/0-387-37825-1

[23] Daneshfar, N. (2021) The Study of Scattering-to-absorption Ratio in Plasmonic Nanoparticles for Photovoltaic Cells and Sensor Applications. Plasmonics 16, 2017-2023. https://doi.org/10.1007/s11468-021-01464-z 
[24] Suarez-Forero, D. G., Riminucci, F. , Ardizzone, V. , Karpowicz, N., Maggiolini, E. , Macorini, G., Lerario, G. , Todisco, F. , De Giorgi, M. , Dominici, L. , Ballarini, D., Gigli, G., Lanotte, A. S., West, K. , Baldwin, K. , Pfeiffer, L. , Sanvitto, D. (2021) Enhancement of Parametric Effects in Polariton Waveguides Induced by Dipolar Interactions. Phys. Rev. Lett. 126, 13740 https://doi.org/10.1103/PHYSREVLETT.126.137401/ FIGURES/4/MEDIUM

[25] Yazdi, H. F., Ghasemi, G., Mohseni, M. , Mohseni, M. (2021) Tuning the dynamics of magnetic droplet solitons using dipolar interactions. Phys. Rev. B 103, 02444. https://doi.org/10.1103/PHYSREVB.103.024441/ FIGURES/6/MEDIUM

[26] Song, X., Dereshgi, S. A., Palacios, E., Xiang, Y. , Aydin, K. (2021) Enhanced Interaction of Optical Phonons in h-BN with Plasmonic Lattice and Cavity Modes. ACS Appl. Mater. Interfaces https://doi.org/10. 1021/ACSAMI.1C00696/SUPPL_FILE/AM1C00696_SI_001.PDF

[27] Feres, F. H., Mayer, R. A. , Wehmeier, L., Maia, F. C.B., Viana, E. R., Malachias, A. , Bechtel, H. A., Klopf, J. M., Eng, L. M., Kehr, S. C., Gonzalez, J. C. Freitas, R. O. Barcelos, I. D. (2021) Sub-diffractional cavity modes of terahertz hyperbolic phonon polaritons in tin oxide. Nat. Commun. 2021121 12, 1-9. https://doi.org/10.1038/s41467-021-22209-w 